\title{
Prioridades de Investigación en Salud en Colombia: Perspectiva de los Investigadores
}

\author{
Health research priorities in Colombia from the researchers' \\ point of view
}

\author{
Carlos A. Agudelo C ${ }^{1}$, Fernando de la Hoz ${ }^{1}$, Martha J. Mojica' ${ }^{1}$, Juan Carlos Eslava \\ $\mathrm{C}^{1}$, Rocío Robledo $\mathrm{M}^{1}$., Patricia Cifuentes ${ }^{1}$ y Nelson Alvis ${ }^{2}$ \\ 1 Instituto de Salud Pública. Departamento de Salud Pública. Facultad de Medicina, Universidad Nacio- \\ nal de Colombia. Bogotá. caagudeloc@unal.edu.co, fpdelahozr@unal.edu.co, jceslavac@unal.edu.co, \\ rrobledom@unal.edu.co \\ 2 Universidad de Cartagena, Colombia. nalvis@yahoo.com
}

Recibido 11 Noviembre 2008/Enviado para Modificación 15 Diciembre 2008/Aceptado 4 Marzo 2009

\section{RESUMEN}

Colombia tiene una escasa experiencia en identificar prioridades de investigación en salud. En el año 2004 se inició un proyecto para identificar prioridades de investigación en salud, entendiendo por tales las que resultan de un ejercicio ordenado de ponderación basado en una valoración juiciosa de problemáticas sanitarias cuya respuesta y/o solución puede lograrse en gran parte por medio de conocimientos y procesos de investigación. Como referentes del proyecto se tuvieron en cuenta algunos de los métodos de priorización utilizados y recomendados en el ámbito internacional, entre ellos la matriz combinada del Global Forum for Health Resarch. Se pusieron en práctica dos trayectos metodológicos principales: por una parte, diseño y aplicación de un método para ponderar u ordenar, de manera cualitativa y cuantitativa, las problemáticas de investigación en salud; por otra parte, construcción de consensos con investigadores y representantes de comunidades científicas. Para identificar las problemáticas de salud predominantes se realizaron dos reuniones nacionales, dos reuniones regionales y un foro virtual. Una vez identificadas las problemáticas de salud predominantes, con su respectiva estimación de carga de enfermedad, estas se valoraron por políticos y decisores y se calificaron por investigadores de ciencias básicas, ciencias clínicas y salud pública, en términos del aporte del conocimiento requerido para afrontar, controlar o resolver tales problemáticas. Se obtuvieron unas prioridades de investigación en salud por áreas globales: enfermedades crónicas, enfermedades infecciosas emergentes, Tuberculosis/Lepra, infección nosocomial e infecciones de transmisión sexual/VIH/SIDA.

Palabras Clave: Agenda de prioridades en salud, prioridades de investigación, investigación, Colombia (fuente: DeCS, BIREME). 


\begin{abstract}
Colombia lacks experience in identifying health research priorities. A project for idenifying health research priorities was begun in 2004 (meaning those arising from weighting and ordering health and disease problems which could be mainly resolved by research and knowledge). The Global Forum for Health Research combined matrix method, and other methods, was used as reference for developing projects and putting two main methodological paths into practice: designing and applying a method for qualitatively and quantitatively weighting and ordering health research problems and building consensus with researchers and scientific community representatives. Two national meetings, two regional meetings and a virtual forum were held for identifying predo-minant health problems. Once the predominant health problems had been identified (with the respective estimation of disease load), then they were evaluated by politi-cians and decision-makers and rated by basic science, clinical science and public health researchers in terms of making a contribution towards knowledge for facing, controlling or resolving such problems. Some health research priorities were obtained (by areas and others being overall priorities): chronic diseases, emergent infectious diseases, tuberculosis/leprosy, nosocomial infection and sexually transmitted diseases/HIV/AIDS.
\end{abstract}

Key Words: Health priorities, research, Colombia (fuente: Mesh, NLM).

$\mathrm{E}$ n la última década se han incrementado los grupos de investigación en Colombia, así como los grupos dedicados a la investigación en salud. De igual manera, han aumentado los recursos destinados a la investigación en salud debido a la creación del Fondo de Investigación en Salud que se nutre del 7 \% de lo producido por las loterías departamentales y los juegos de azar. Estos recursos han permitido aumentar el número de proyectos en Salud financiados por año y la diversificación de la investigación en salud. Sin embargo, persisten desequilibrios entre la carga de las problemáticas de en-fermedad y los recursos de investigación que se dedican a las mismas (1). Problemas como las lesiones por causa violenta, la enfermedad respiratoria aguda y crónica, los problemas perinatales, los problemas nutricionales y las enfermedades congénitas, causan el 56\% de la carga de enfermedad en el país, pero en los 4 años anteriores al 2005, el Fondo de Investigación en Salud, solo ha dedicado el 10\% de sus recursos a financiar investigación en tales problemáticas. Por el contrario, a las enfermedades infecciosas que causan alrededor del $25 \%$ de la carga de enfermedad, se ha dedicado el $47 \%$ de los recursos de este Fondo.

Pese a que el desequilibrio no es tan dramático como el que existe a nivel mundial (2), es necesario implementar medidas para fomentar la investiga-ción en salud en aquellos problemas que causan una proporción importante de carga de enfermedad, con el fin de que la investigación contribuya de manera más eficaz al desarrollo humano. 


\section{Aspectos teóricos y metodológicos}

En salud pública se suelen utilizar al menos tres tipos de escenarios para identificar prioridades y asignar recursos de acuerdo con ellas: atención a la salud (3), problemáticas e intervenciones en salud (4) e investigación en salud (5). En los tres escenarios se parte de la misma pregunta: ¿Para solucionar las problemáticas y necesidades (siempre amplias) que afectan la salud y la calidad de vida de la población, dónde o en qué se requiere concentrar los esfuerzos y los recursos (siempre limitados)? Sin embargo, el alcance de los escenarios es distinto. En el primer escenario, la prioridad de los esquemas de atención a las personas intenta equilibrar eficiencia, eficacia, cobertura, costos y financiación desde el punto de vista de la equidad y la justicia social. En el segundo, las prioridades intentan delimitar aquellas respuestas o intervenciones que pueden contribuir al mejoramiento de la salud de la población general o específica, teniendo en cuenta cómo se distribuye la salud y la enfermedad en la población, las preferencias sociales y los recursos disponibles. Los dos esquemas de priorización no son excluyentes sino complementarios, porque suponiendo que se han asignado los recursos a la atención en salud de manera equitativa y eficiente, aún se requerirán intervenciones específicas para responder a problemas que se concentran en ciertos momentos o grupos de la población o en regiones específicas.

Para la identificación de prioridades en los dos casos anteriores se utilizan criterios como la medicina basada en la evidencia, análisis de carga de enfermedadAVISAS, análisis de costo-efectividad y análisis de equidad (4). A pesar de las numerosas metodologías y aproximaciones desarrolladas para priorizar, con frecuencia se aplica sólo uno de los criterios mencionados, mientras que los decisores y políticos esperan aproximaciones que tomen en cuenta criterios múltiples para responder a realidades complejas. Téngase en cuenta que aquellos están, a su vez, en conexión con actores cuyos intereses o perspectivas son diferentes y aun contradictorios (autoridades políticas, instituciones, proveedores de atención, pacientes, usuarios, etc.). Por esta razón, los esquemas de priorización se encuentran en medio de tensiones potentes, entre quienes los definen y aquellos a quienes supuestamente les son útiles o benefician.

Las prioridades de investigación en salud también tienen como referente las problemáticas y necesidades de la población. Pero el enfoque para priorizar en investigación difiere de manera significativa con respecto a los métodos ya comentados. Aunque ninguna problemática o necesidad de salud de la población puede ser resuelta únicamente con investigación, ya que además se requieren inversiones, planes, administración y otros aspectos similares, se consideran o califican 
como prioridades de investigación, aquellas problemáticas en las que el conocimiento aporta de manera significativa a su solución, control o manejo. En otras palabras, las prioridades de investigación en salud no se desprenden únicamente de la magnitud, gravedad o relevancia de las problemáticas y necesidades. Adicionalmente, se requiere esclarecer si se trata, y en qué grado, de problemas de investigación, o sea, problemas para ser resueltos en gran parte por medio de conocimientos nuevos. Adicionalmente, es imprescindible establecer si existen las condiciones materiales y humanas para investigar y producir tal conocimiento.

En Colombia se cuenta con una experiencia amplia en la identificación de prioridades en términos de la atención a la salud (por ejemplo, el modelo del Plan Obligatorio de Salud-POS); se cuenta, así mismo, con experiencias diversas en términos de intervenciones, sin esquemas explícitos de prioridades. El Ministerio de la Protección Social y el Plan Nacional de Salud Pública (6) describen unas "Prioridades Nacionales de Salud" entendidas como respuesta a las necesidades de salud, teniendo en cuenta los recursos disponibles. No son, por tanto, en sentido estricto, prioridades de investigación.

Por otra parte, hay muy poca experiencia en prioridades de investigación en salud, a pesar de que son relativamente conocidos los métodos de priorización en salud reconocidos en el ámbito internacional. Hace más de dos décadas se han planteado propuestas dirigidas a estimular los procesos para identificar prioridades de investigación en salud en los países en desarrollo. Algunos de los métodos más utilizados, desde 1987, son el de la "investigación nacional esencial en salud" del COHRED $(7,8)$, el método de los cinco pasos (9) y, el método de la matriz combinada (2). Estos tres métodos aplican criterios múltiples.

Prioridades de investigación en salud en Colombia

A finales del año 2004 Colciencias y el Instituto de Salud Pública de la Universidad Nacional de Colombia (con el apoyo del Global Forum for Health Resarch), acordaron realizar un proyecto de construcción de una agenda de prioridades de investigación en salud. Si bien participaron un conjunto de instituciones públicas y privadas (Asociación de Municipios, Sociedades Científicas, Empresas Promotoras de Salud-EPS e Instituciones Prestadoras de Servicios-IPS), el proceso estuvo centrado en la participación y la pers-pectiva de los investigadores en salud, de las áreas de ciencias básicas, clínicas y salud pública.

Trayectos Metodológicos. Se interpretó el ejercicio de priorización como la ponderación y ordenamiento de problemáticas de salud y enfermedad cuya respuesta 
y/o solución puede lograrse en gran parte por medio de la generación de nuevos conocimientos y procesos de investigación. Las prioridades de investigación en salud son el resultado de este tipo de ejercicio.

En Febrero de 2005 se realizó la I Reunión Nacional de Construcción de una Agenda de Prioridades de Investigación en Salud. En esta Reunión Nacional se examinaron las tendencias de la mortalidad en Colombia de 1997 a 2001, las prioridades del Ministerio de la Protección Social, la situación de la investigación en salud en Colombia y el direccionamiento estratégico de Colciencias. Como aspecto central de la Reunión, los investigadores y grupos de trabajo identificaron temas prioritarios (problemas de salud y enfermedad del país) de acuerdo con su experiencia, en tres áreas: ciencias básicas, ciencias clínicas y salud pública.

Posteriormente a la I Reunión Nacional la lista de necesidades, temáticas y problemáticas de salud-enfermedad del país, planteada por los investigadores, se comparó con la lista de prioridades de Ministerio de la Protección Social y se elaboró una lista unificada de problemáticas nacionales relevantes. Se utilizaron dos procedimientos adicionales para enriquecer la lista unificada:

- A cada una de las problemáticas identificadas se le adjuntó la información disponible sobre carga de enfermedad, verificando su importancia con este tipo de indicador.

- Las problemáticas fueron examinadas por tres políticos y/o decisores. Cada uno de ellos hizo el ejercicio de indicar la importancia que aquellas tendrían como parte de la política pública y la gestión de programas de salud.

Métodos de ponderación de problemáticas. Con base en la lista unificada y su propia experiencia, los investigadores identificaron el grado en que aporta la investigación y el conocimiento al control o solución de los problemas de salud-enfermedad relevantes en el país, desde las ciencias básicas, las ciencias clínicas y la salud pública. Para ello, mediante el trabajo de grupos y el consenso, se aplicaron a las problemáticas de la lista unificada, los criterios cualitativos y su expresión cuantitativa o puntaje, que se indican a continuación.

\begin{tabular}{cc}
\hline $\begin{array}{c}\text { Necesidad de } \\
\text { conocimiento para el } \\
\text { control del problema }\end{array}$ & Puntaje individual \\
\hline Ninguna & 0 \\
Muy poca & 1 \\
Intermedia & 2 \\
Alta & 3 \\
\hline
\end{tabular}


Por este medio se obtuvieron listas o tablas de prioridades en cada área y luego una apreciación global, mediante la agregación de los puntajes.

A partir de la I Reunión Nacional, el proceso anterior se llevó a cabo por medio de cuatro actividades centrales.

- Diseño, organización y puesta en funcionamiento de un Foro Virtual para examinar los aspectos metodológicos de la priorización de la investigación en salud (10). El Foro Virtual se realizó de Julio a Diciembre de 2005.

- Organización de la II Reunión Nacional de Prioridades de Investigación en Salud, en Bogotá (11). Esta reunión se realizó en Diciembre de 2005.

- Organización y realización de dos Reuniones Regionales, en Cartagena (Junio de 2006) y Manizales (Julio de 2006).

En estas reuniones se hizo el ejercicio de ponderar e identificar prioridades de investigación, por medio del método ya indicado. En estas actividades participaron un total de 160 investigadores de las ciencias básicas, clínicas y salud públicaciencias sociales, de los cuales 21 eran del exterior.

Resultados. A continuación presentamos únicamente las cinco primeras opciones o prioridades por áreas y la ubicación global obtenidas en cada una de las reuniones.

En estas tablas se observan unas ciertas regularidades en la ubicación de las problemáticas por áreas y en las apreciaciones globales. Dado que se aplicó la misma metodología de ponderación en las tres reuniones, es posible considerar de conjunto los puntajes por áreas para obtener una valoración más amplia. Para ello, se conformó un solo grupo por área con las problemáticas de las tres reuniones. Se interpretaron como primer lugar aquellas problemáticas que se ubicaron entre las cinco primeras preferencias en las tres reuniones. Se interpretaron como segundo lugar aquellas problemáticas que se ubicaron entre las cinco primeras preferencias de los investigadores, en dos de las tres reuniones. El resultado de este ejercicio por áreas puede ser inter-pretado como un esquema de prioridades (Tabla 4). 
Tabla 1. Resultados obtenidos en la II Reunión Nacional de prioridades de investigación

\begin{tabular}{|c|c|c|c|c|c|c|c|}
\hline \multicolumn{2}{|l|}{ Básica } & \multicolumn{2}{|l|}{ Clínica } & \multicolumn{2}{|c|}{ S. Pública } & \multicolumn{2}{|l|}{ Global } \\
\hline Prob & $\mathrm{P}$ & Prob & $\mathrm{P}$ & Prob & $\mathrm{P}$ & Prob & $\mathrm{P}$ \\
\hline $\begin{array}{c}\text { Enf inf. } \\
\text { Emergentes }\end{array}$ & 23 & Enf crónicas & 23 & $\begin{array}{c}\text { Salud } \\
\text { Materna }\end{array}$ & 27 & Enf crónicas & 63 \\
\hline TBC/lepra & 20 & Salud mental & 21 & $\begin{array}{c}\text { Violencia } \\
\text { Intrafamiliar }\end{array}$ & 27 & $\begin{array}{c}\text { Enf inf. } \\
\text { Emergentes }\end{array}$ & 60 \\
\hline $\begin{array}{l}\text { Trasmit } \\
\text { vectores }\end{array}$ & 17 & Polit Farmac & 21 & $\begin{array}{c}\text { Salud } \\
\text { pública }\end{array}$ & 27 & $\begin{array}{c}\text { Trasmit } \\
\text { vectores }\end{array}$ & 58 \\
\hline Inf Nosocom & 16 & $\begin{array}{c}\text { Enf inf. } \\
\text { Emergentes }\end{array}$ & 21 & Enf crónicas & 26 & TBC/lepra & 58 \\
\hline ITS/VIH/SIDA & 15 & $\begin{array}{c}\text { Trasmit } \\
\text { vectores }\end{array}$ & 20 & $\begin{array}{c}\text { Prestación } \\
\text { servicios }\end{array}$ & 26 & Polit Farmac & 58 \\
\hline
\end{tabular}

Prob: Problemáticas; P: puntaje

Tabla 2. Reunión de la Costa Atlántica de prioridades de investigación

\begin{tabular}{|c|c|c|c|c|c|c|c|}
\hline \multicolumn{2}{|l|}{ Básica } & \multicolumn{2}{|l|}{ Clínica } & \multicolumn{2}{|c|}{ Salud Pública } & \multicolumn{2}{|l|}{ Global } \\
\hline Prob & $\mathrm{P}$ & Prob & $\mathrm{P}$ & Prob & $\mathrm{P}$ & Prob & $\mathbf{P}$ \\
\hline ITS $N I H / S I D A$ & 34 & ITS/VIH/SIDA & 33 & Salud Materna & 44 & ITS/VIH/SIDA & 107 \\
\hline Enf crónicas & 32 & Enf crónicas & 32 & $\begin{array}{c}\text { Prestación } \\
\text { serv }\end{array}$ & 43 & Enf crónicas & 105 \\
\hline TBC/lepra & 30 & $\begin{array}{l}\text { Salud } \\
\text { Materna }\end{array}$ & 32 & $\begin{array}{l}\text { Salud Pob } \\
\text { Vulner }\end{array}$ & 43 & $\begin{array}{l}\text { Salud } \\
\text { Materna }\end{array}$ & 98 \\
\hline $\begin{array}{c}\text { Enf infecciosas } \\
\text { Emergentes }\end{array}$ & 27 & $\begin{array}{c}\text { Inf } \\
\text { Nosocomial }\end{array}$ & 31 & $\begin{array}{c}\text { Viol } \\
\text { Intrafamiliar }\end{array}$ & 42 & $\begin{array}{c}\text { Infección } \\
\text { Nosocomial }\end{array}$ & 90 \\
\hline $\begin{array}{l}\text { Enf trasmitidas } \\
\text { por vectores }\end{array}$ & 27 & IRA/EDA & 27 & $\begin{array}{l}\text { Enfermedades } \\
\text { crónicas }\end{array}$ & 41 & $\begin{array}{c}\text { Enf } \\
\text { infecciosas } \\
\text { Emergentes }\end{array}$ & 89 \\
\hline
\end{tabular}

Prob: Problemáticas; P: puntaje

Tabla 3. Reunión de Occidente de prioridades de investigación

\begin{tabular}{|c|c|c|c|c|c|c|c|}
\hline \multicolumn{2}{|l|}{ Básicas } & \multicolumn{2}{|l|}{ Clínicas } & \multicolumn{2}{|l|}{ S. Pública } & \multicolumn{2}{|l|}{ Global } \\
\hline Prob & $P$ & Prob & $P$ & Prob & $\mathrm{P}$ & Prob & $P$ \\
\hline $\begin{array}{l}\text { Políticas } \\
\text { Fármacos }\end{array}$ & 24 & $\begin{array}{l}\text { Políticas } \\
\text { Fármacos }\end{array}$ & 27 & $\begin{array}{c}\text { Viola } \\
\text { Intrafamiliar }\end{array}$ & 30 & $\begin{array}{l}\text { Políticas } \\
\text { Fármacos }\end{array}$ & 76 \\
\hline $\begin{array}{c}\text { Enf infecciosas } \\
\text { Emergentes }\end{array}$ & 19 & Salud Materna & 24 & $\begin{array}{c}\text { Enf } \\
\text { trasmitidas } \\
\text { por vectores }\end{array}$ & 30 & ITS/VIH/SIDA & 68 \\
\hline $\begin{array}{c}\text { Infección } \\
\text { Nosocomial }\end{array}$ & 18 & $\begin{array}{l}\text { Enfermedades } \\
\text { crónicas }\end{array}$ & 24 & Salud pública & 30 & $\begin{array}{l}\text { Enfermedades } \\
\text { crónicas }\end{array}$ & 68 \\
\hline ITS/VIH/SIDA & 16 & ITS/VIH/SIDA & 23 & ITS/NIH/SIDA & 29 & $\begin{array}{l}\text { Infección } \\
\text { Nosocomial }\end{array}$ & 65 \\
\hline $\begin{array}{l}\text { Enfermedades } \\
\text { crónicas }\end{array}$ & 16 & $\begin{array}{c}\text { Infección } \\
\text { Nosocomial }\end{array}$ & 23 & IRA/EDA & 29 & TBC/lepra & 64 \\
\hline
\end{tabular}

Prob: Problemáticas; P: puntaje

Una última lista de cinco preferencias resulta de la confluencia de los puntos de vista de los investigadores en las tres áreas, en el sentido de aquellas problemáticas en las que la investigación puede aportar significativamente a su control o resolución en todas las áreas (Tabla 4, columna derecha). 
Tabla 4. Prioridades por áreas

\begin{tabular}{|c|c|c|c|c|}
\hline Posición & Ciencias básicas & Ciencias Clínicas & Salud Pública & Todas I as áreas \\
\hline $\begin{array}{l}\text { Primer } \\
\text { lugar }\end{array}$ & $\begin{array}{l}\text { Enf. infecciosas } \\
\text { emergentes }\end{array}$ & $\begin{array}{l}\text { Enfermedades } \\
\text { crónicas }\end{array}$ & $\begin{array}{l}\text { Violencia } \\
\text { intrafamiliar }\end{array}$ & $\begin{array}{l}\text { Enfermedades } \\
\text { crónicas }\end{array}$ \\
\hline \multirow[t]{4}{*}{$\begin{array}{l}\text { Segundo } \\
\text { lugar }\end{array}$} & $\begin{array}{l}\text { Enf. Transmitidas } \\
\text { por vectores }\end{array}$ & $\begin{array}{l}\text { Política } \\
\text { farmacéutica }\end{array}$ & $\begin{array}{l}\text { Salud Pública } \\
\text { (SGSSS) }\end{array}$ & $\begin{array}{l}\text { Enfermedades } \\
\text { infecciosas } \\
\text { emergentes }\end{array}$ \\
\hline & $\begin{array}{l}\text { Infecciones } \\
\text { Nosocomiales }\end{array}$ & ITS/VIH/SIDA & $\begin{array}{l}\text { Enfermedades } \\
\text { Crónicas }\end{array}$ & TBC/Lepra \\
\hline & TBC/Lepra & Salud materna & Salud materna & $\begin{array}{l}\text { Infección } \\
\text { nosocomial }\end{array}$ \\
\hline & & $\begin{array}{l}\text { Infección } \\
\text { nosocomial }\end{array}$ & $\begin{array}{l}\text { Prestación de } \\
\text { servicios }\end{array}$ & ITS/VIH/SIDA \\
\hline
\end{tabular}

\section{Criterios de priorización}

La investigación en salud es un ejercicio de lógicas complementarias y su agenda de prioridades no corresponde exactamente a la agenda de necesidades de salud de la población

La experiencia permitió probar y aplicar parcialmente los criterios de priorización del método de la matriz combinada, así como valorar otros criterios para priorizar la investigación en salud en nuestras condiciones, en el nivel nacional y regional. Aunque todos ellos deben ser sometidos a nuevas pruebas, cabe mencionar los de tipo central: Problemáticas de salud-enfermedad, determinantes de la salud, factores de riesgo, sistemas y políticas de salud, alcance y eficacia de los conocimientos y tecnologías actuales y disponibles, percepción política de los decisores y costo efectividad de las intervencio-nes. Así mismo, debe mencionarse los criterios de contexto y proceso: Politicas nacionales y regionales de Ciencia y Tecnología de la salud, así como la disponibilidad y el flujo de recursos para investigar en salud; Articulación y construcción de consensos con comunidades, grupos de interés, ONG, instituciones, etc. (sociedad civil, comunidades, usuarios, consejos territo-riales, etc).

Como limitación, el proceso realizado fue sólo un paso en la construcción de una agenda de prioridades de investigación en salud.

Desde un punto de vista general, la construcción de agendas de prioridades de investigación en salud es un proceso político explícito que requiere con-sensos, en la medida que involucra múltiples actores, con experiencias y lenguajes diferentes, tanto en investigación como en salud. Este tipo de ejercicios para establecer prioridades explícitas de investigación permite responder de qué manera contribuye nuestro trabajo de investigadores en salud al 
desarrollo humano. No sólo fortalece a las comunidades científicas, sin que también asegura el mejor uso de los recursos disponibles, desde valores como la equidad y refuerza los vínculos entre investigación y política. Adicionalmente, mejora la eficacia de las políticas y programas de fomento a la investigación, facilitando afrontar los desafíos de coordinación y articulación. Las prioridades de investigación enlazan necesidades de salud de la población y políticas de salud y de investigación. Por último, las prioridades abren una perspectiva estratégica que permite distanciarse de una visión reduccionista (sólo importan los conocimientos básicos), de una visión lineal (únicamente de la acumulación de conocimiento básico se deriva lo tecnológico) y de una visión utilitarista (sólo importa lo que es aplicable)

\section{REFERENCIAS}

1. Universidad Nacional de Colombia, Colciencias, Global Forum for Health Research [Internet] .Construcción de una agenda de prioridades de investigación en salud. Memorias. Disponible en: http://www.revmed.unal.edu.co/red/prioridad/MEMORIAS.pdf Consultado: Septiembre 2008.

2. GFHR (Global Forum for Health Research. 2002. The 10/90 Report on Health Research 20012002. Geneva: Global forum for Health Research

3. Sabik LM, Lie RK. Priority setting in health care: Lessons from the experiences of eight countries. International Journal for Equity in Health. 2008; 7:4

4. Baltussen R, Niessen L. Priority setting of health interventions: the need for multi-criteria decision analysis. Cost Effectiveness and Resource Allocation 2006, 4:14

5. Alliance for Health Policy and Systems Research. From Mexico to Mali: Taking stock of achievements in health policy and systems research. Report of Meeting. Nyon, Switzerland. May, 2008. $38 p$.

6. Ministerio de la Protección Social. Decreto 3039 de 2007. «Por el cual se adopta el Plan Nacional de Salud Pùblica 2007-2010", Bogotá, 2007.

7. Advisory Committee on Health Research. 1997. A Research Policy Agenda for Science and Technology to Support Health Development. Geneva: World Health Organization. WHO/ RPS/ACHR/97.3 for Development, COHRED Document No. 2000.3.

8. COHRED (Council on Health Research for Development) 2000. A manual for Research Priority Setting Using the ENHR Strategy. Geneva:Council on Health Research for Develop-ment, COHRED Document No. 2000.3.

9. Ad Hoc Committee (Ad Hoc Committee on Health Research Relating to Future Intervention Options) 1996. Investing in Health Research and Development. Geneva: World Health Organi-zation. TDR/Gen/96.1

10. Prioridades de investigación en Salud. Foro Virtual [Internet] Disponible en: http:// www.revmed.unal.edu.co/red/prioridad/nuevapropuesta1.pdf Consultado: Octubre 2008.

11. Prioridades de Investigación en Salud. II Reunión Nacional. Disponible en: http:// www.revmed.unal.edu.co/red/intro18.htm Consultado: Octubre 2008. 